\title{
Parámetros de crecimiento y adiposidad corporal de adolescentes chilenos a través de la referencia CDC-2000 y CDC-2012: estudio ACECH*
}

\section{Growth parameters and body adiposity in chilean adolescents reference to the CDC-2000 and CDC-2012: ACECH* study}

\section{ABSTRACT}

The aim of the study was to compare physical growth and body adiposity in Chilean adolescents according to the CDC-2000 and CDC- 2012 reference. A number of 19,970 students, between 14 and 18 years old, participated in the study. Adolescents belonged to the three units of the Chilean school education system. Weight, height and Body Mass Index were measured. The results indicated that Chilean students showed similar body weight, low height and high prevalence of overweight (28.3 to $32.5 \%$ ) according to the CDC-2000 reference. However, by the CDC- 2012 reference, results showed low weight and height, and consequently lower values of overweight (9.0 to 6.6\%). We conclude that the use of a reference updates can cause confusion when analyzing the physical growth and body adiposity of Chilean adolescents. The results suggest the need for construction of a national curve which can be used to make the assessment of growth and nutritional status more reliably.

Key words: Growth, adiposity, adolescent, curves for weight and height, Chile.
Rodrigo Vargas V. (1) Cecilia Bahamonde P. (2)

Jorge Cancino L. (3)

Paulina Correa E. (4)

Simón Michelow B. (5)

Patricio Gatica M. (1)

Rossana Gómez C. (6)

Cristian Martínez S. (6)

Diego Vargas V. (7)

Daniela Luna S. (7)

Marco Cossio-Bolaños $(1,8)$

(1) Departamento de Ciencias de la Actividad Física, Universidad Católica del Maule, Talca, Chile. (2) Universidad Metropolitana de Ciencias de la Educación, Santiago, Chile. (3) Facultad de Ciencias de la Actividad Física Universidad San Sebastián, Santiago, Chile. (4) Departamento de investigación y diseño IND, Santiago, Chile. (5) División Actividad Física y Deporte IND, Santiago, Chile.

(6) Departamento Educación Física, Universidad de La Frontera, Temuco, Chile. (7) Facultad de Medicina Universidad Católica del Maule, Talca, Chile. (8)Laboratorio de Rendimiento Humano, Universidad Católica del Maule, Talca, Chile.

*ACECH (Agencia de la Calidad de la Educación Chile).

Dirigir la correspondencia a: Prof. Dr. Marco Cossio-Bolaños Departamento de Ciencias de la Actividad Física Universidad Católica del Maule Av. San Miguel 3605, Talca, Chile Teléfono: 071-2203447 E-mail: mcossio1972@hotmail.com

Este trabajo fue recibido el 24 de Septiembre de 2013 y aceptado para ser publicado el 18 de Enero de 2014.

\section{INTRODUCCIÓN}

La determinación del crecimiento físico tiene diversas aplicaciones, tales como detectar la situación actual del individuo en cuanto a los patrones nutricionales, valorar las prácticas de introducción a la alimentación complementaria, además de realizar un seguimiento a grupos con riesgo nutricional (1). Por su parte la adiposidad corporal es fundamental para la prevención de enfermedades y la promoción de la salud (2), sobre todo, si el término adiposidad se usa para analizar el exceso de peso, puesto que la distribución de grasa corporal ha sido asociada significativamente como elemento de predicción del factor de riesgo de enfermedades cardiovasculares en adultos (3), en niños y adolescentes de diversas regiones del mundo $(4,5)$.

En general, la valoración del crecimiento físico y la adiposidad corporal que engloban estudios de grandes poblaciones se realizan a menudo a través de medidas antropométricas. De hecho, para el crecimiento se utilizan variables clásicas de 
peso y estatura $(6,7)$ y para la adiposidad corporal el Índice de Masa Corporal (IMC), pliegues cutáneos y perímetros de diversas regiones corporales (8).

Actualmente existen varias referencias internacionales que permiten interpretar las variables del crecimiento físico en peso y estatura $(9,10)$, así como del perímetro de la cintura $(11,12)$ y el Índice de masa Corporal para el caso de la adiposidad corporal $(10,12,13)$. Tales referencias permiten valorar el crecimiento físico, estado nutricional y la adiposidad corporal de niños y adolescentes de forma separada, lo que impide el uso de una sola referencia para valorar las tres variables en un sólo momento. En este sentido, recientemente el Centro para el Control y la Prevención de Enfermedades CDC-2012 (12) ha publicado nuevas normas que abarcan variables de peso, estatura, pliegues cutáneos, circunferencias y longitudes corporales que permiten valorar y realizar el seguimiento del crecimiento físico, estado nutricional y adiposidad corporal de niños y adolescentes a través de una misma referencia. Estas curvas presentan información actualizada respecto a otras referencias internacionales, inclusive, en relación al CDC2000 (10). Desde esa perspectiva, estudiar los parámetros del crecimiento físico y la adiposidad corporal de adolescentes escolares a través de referencias internacionales sin previa actualización, probablemente no permita monitorizar de forma adecuada los patrones de crecimiento físico e identificar y clasificar el exceso de peso corporal de adolescentes chilenos. En este sentido, este estudio buscó comparar los parámetros del crecimiento físico y la adiposidad corporal de adolescentes chilenos en función de la referencia del CDC2000 y CDC-2012.

\section{SUJETOS Y MÉTODOS}

Tipo de investigación y muestra

El estudio es de tipo descriptivo comparativo. La muestra comprende 19770 escolares adolescentes, siendo 13580 hombres y 6390 mujeres, cuyas edades oscilan entre los 14 a 18 años de edad. Los escolares fueron seleccionados de forma no-probabilística por cuotas. La recolección de datos se efectuó durante el mes de noviembre del 2011 en 15 regiones de Chile. El estudio abarca tres dependencias del sistema educativo chileno (centros municipalizados, particulares subvencionados y particulares pagados) y tres niveles socioeconómicos (bajo, medio y alto). La tabla 1 muestra las características de la muestra estudiada.
En general, todos los alumnos que se sometieron a la evaluación del SIMCE realizaban actividad física de 2 veces por semana, en el primer día 90 minutos y en el segundo día 60 minutos, totalizando 150 minutos /semana de actividad física de moderada intensidad.

La condición socioeconómica se midió por medio de la metodología propuesta y desarrollada por el Sistema de Medición de la Calidad de la Educación del Ministerio de Educación de Chile SIMCE (14), donde es posible distinguir a los escolares en categorías: bajo, medio y alto. Se consideró como criterios de inclusión a los escolares de las tres categorías antes indicadas y a los que firmaron el consentimiento informado para la realización de medidas antropométricas. Fueron excluidos los escolares (28 escolares), cuyos apoderados y/o padres no autorizaron las mediciones antropométricas de sus hijos y los que presentaban problemas motores y dificultades físicas. El estudio se realizó de acuerdo a los principios de Helsinki y contó con la debida aprobación del Comité de Etica.

Técnicas y procedimientos

La edad decimal se calculó a partir del registro de la fecha de nacimiento (día, mes y año) y la fecha de valoración antropométrica (día, mes y año). El cálculo se efectuó por medio de una tabla de registros, a través, del cual se determinó la edad decimal. Las medidas antropométricas fueron controladas a través de técnicas y protocolos estandarizados. Se adoptó las sugerencias descritas por el Centro Nacional de Estadísticas de Salud y el Centro para el Control y la Prevención de Enfermedades (NHANES) (15).

El peso corporal se midió con una balanza de marca Tanita con precisión de 0,1 $\mathrm{Kg}$. La estatura se evaluó utilizando un estadiómetro de aluminio con precisión de $0,1 \mathrm{~cm}$. Todas las variables antropométricas valoradas fueron efectuadas por profesionales de educación física con amplia experiencia en recolección de variables antropométricas. Los mismos antes de realizar la toma de datos se sometieron a una capacitación para efectuar la medición de variables antropométricas, el cual estuvo a cargo de académicos especialistas de las universidades: Católica del Maule, Metropolitana de Ciencias de la Educación y Universidad Mayor.

El Índice de Masa Corporal $\left(\mathrm{kg} / \mathrm{m}^{2}\right)$ fue calculado a través de la relación del peso corporal con la estatura al cuadrado. Se utilizó la fórmula propuesta por Quetelet (16) donde $\left[\mathrm{IMC}=\operatorname{Peso}(\mathrm{kg}) /\right.$ Estatura $\left.(\mathrm{m})^{2}\right]$.

TABLA 1

Características de la muestra estudiada.

\begin{tabular}{lcc} 
Variables & $\mathrm{fi}$ & $\%$ \\
Sexo: & & 32,0 \\
Masculino & 6390 & 68,0 \\
Femenino & 1358 & \\
Dependencia: & & 45,4 \\
Municipal & 9071 & 46,7 \\
Subvencionado & 9333 & 7,8 \\
Pagado & 1566 & 12,1 \\
Condición socioeconómica: & & 65,8 \\
Baja & 2412 & 22,1 \\
Media & 13151 & 4407 \\
Alta & & \\
\hline
\end{tabular}


Para determinar la prevalencia de obesidad, sobrepeso y normalidad se utilizó como criterio el IMC, cuyos puntos de corte corresponden a la CDC-2000 (10) y CDC-2012 (12): bajo $\langle p 10$, eutrófico $p>=10-p>=85$, sobrepeso $p<=85-p<95$ y obeso $p>=95$.

\section{Análisis estadístico}

Los datos fueron normalizados por el test de KolmoronovSmirnov. Los resultados fueron analizados a partir de estadígrafos descriptivos (media, desviación estándar y porcentajes). Las diferencias entre sexos se verificaron a partir del test t para muestras independientes. Las comparaciones entre el estudio y la referencia se determinaron por medio del test $t$ para muestras pareadas (Tukey). La prevalencia de adiposidad fue comparada por medio del test de $\mathrm{X}^{2}$ según sexo y fase etaria. En todos los análisis estadísticos se adoptó una significancia de $p<0,001$.

\section{RESULTADOS}

Los valores medios y error estándar de las variables antropométricas y del Índice de Masa Corporal (IMC) para ambos sexos se observan en la tabla 2. Todas las variables antropométricas aumentan conforme la edad avanza. Los hombres son más altos que las mujeres a todas las edades, a su vez, son más pesados a partir de los 16 años en adelante. Respecto a la adiposidad corporal los hombres presentan un IMC mayor en relación a las mujeres $(p<0,001)$.

La figura 1 ilustra las diferencias de peso y estatura entre las dos referencias del CDC (2000 y 2012) con el estudio. En relación al peso corporal no hubo diferencias en ambos sexos respecto a la referencia del CDC-2000, pero si observamos diferencias significativas respecto a la referencia del CDC-2012 en todas las edades; sin embargo, en cuanto a la estatura, ambas referencias muestran valores superiores en comparación con el estudio.

La figura 2 muestra los valores medios y desviación estándar del Índice de Masa Corporal (IMC) del estudio con la referencia del CDC-2000 y CDC-2012. En general, se ob- servan diferencias con la referencia del CDC-2000 en ambos sexos. Sin embargo, cuando se comparó con el CDC-2012 se determinó diferencias significativas en las mujeres a los 14 y 15 años y en los hombres desde los 15 hasta los 18 años, respectivamente.

La tabla 3 muestra los valores medios y $\pm \mathrm{DE}$ Índice de Masa Corporal (IMC) y la prevalencia de bajo peso y exceso de peso de adolescentes chilenos (diagnosticada a partir del IMC). Los resultados muestran que no hubo diferencias en el IMC en adolescentes de ambos sexos. Por otro lado, en relación a la prevalencia del bajo y exceso de peso, los resultados evidencian diferencias entre los valores obtenidos a través del CDC-2000 y CDC-2012 en adolescentes de ambos sexos. En general, la referencia del CDC-2012 permite clasificar mayor cantidad de individuos en rangos de normalidad en relación al CDC-2000.

\section{DISCUSIÓN \\ Crecimiento físico}

Los resultados del estudio evidencian que los adolescentes de ambos sexos considerados en la aplicación SIMCE presentaron valores medios similares de peso corporal en relación a la referencia del CDC-2000, sin embargo, respecto a los valores medios de la referencia del CDC-2012, los adolescentes del estudio presentan valores inferiores. Evidentemente estos hallazgos podrían deberse a factores relacionados con la tendencia secular, dado que las curvas recientes del CDC-2012 fueron propuestos con la intención de mejorar el conocimiento acerca del crecimiento y desarrollo del niño y del adolescente Americano (12). De hecho, todo estándar debe ser actualizado a lo largo del tiempo, puesto que los profundos cambios sociales y económicos ocurridos en todo el mundo en las últimas décadas se han acompañado de un aumento en el tamaño corporal (17), inclusive, los patrones dietéticos han cambiado en su mayor parte (18) aumentando el consumo de nutrientes ricos en grasas. En este sentido, era de esperar que el peso corporal de los adolescentes estudiados estuviera por encima de la

TABLA 2

Tamaño de la muestra y estadística descriptiva de las variables antropométricas de adolescentes chilenos.

\begin{tabular}{|c|c|c|c|c|c|c|c|}
\hline \multirow[t]{2}{*}{ Edad (años) } & \multirow[b]{2}{*}{$\mathrm{n}$} & \multicolumn{2}{|c|}{ Masa corporal (Kg) } & \multicolumn{2}{|c|}{ Estatura (cm) } & \multicolumn{2}{|c|}{$\mathrm{IMC}\left(\mathrm{Kg} / \mathrm{m}^{2}\right)$} \\
\hline & & x & $\mathrm{EE}$ & $x$ & $\mathrm{EE}$ & $x$ & EE \\
\hline \multicolumn{8}{|c|}{ Mujeres $(\mathrm{n}=6390)$} \\
\hline 14 & 2218 & 55,19 & 0,18 & 156,4 & 0,1 & 22,52 & 0,07 \\
\hline 15 & 3532 & 56,26 & 0,14 & 157,4 & 0,08 & 22,67 & 0,05 \\
\hline 16 & 456 & 57,46 & 0,41 & 156,7 & 0,22 & 23,35 & 0,15 \\
\hline 17 & 106 & 60,56 & 0,92 & 157 & 0,45 & 24,45 & 0,35 \\
\hline 18 & 78 & 60,81 & 1,13 & 156,8 & 1,03 & 24,55 & 0,77 \\
\hline \multicolumn{8}{|c|}{ Hombres $(n=13580)$} \\
\hline 14 & 4385 & 55,93 & 0,11 & 158,9 & $0,07^{*}$ & 22,09 & $0,04^{*}$ \\
\hline 15 & 7387 & 57,66 & 0,11 & 161,1 & $0,07^{*}$ & 22,17 & $0,04^{*}$ \\
\hline 16 & 1305 & 60,71 & $0,27^{*}$ & 163,5 & $0,20^{*}$ & 22,7 & $0,09 *$ \\
\hline 17 & 395 & 63,06 & $0,54^{*}$ & 164,5 & $0,37^{*}$ & 23,17 & $0,19^{*}$ \\
\hline 18 & 108 & 62,98 & $1,14^{*}$ & 164,6 & $0,73^{*}$ & 23,21 & $0,38^{*}$ \\
\hline
\end{tabular}

* $(p<0,001)$ : Diferencia significativa en relación a las mujeres. 


\section{FIGURA 1}

Variables de crecimiento físico del estudio, CDC-2000 y CDC-2012 expresados en promedio y Error Estándar de adolescentes chilenos.
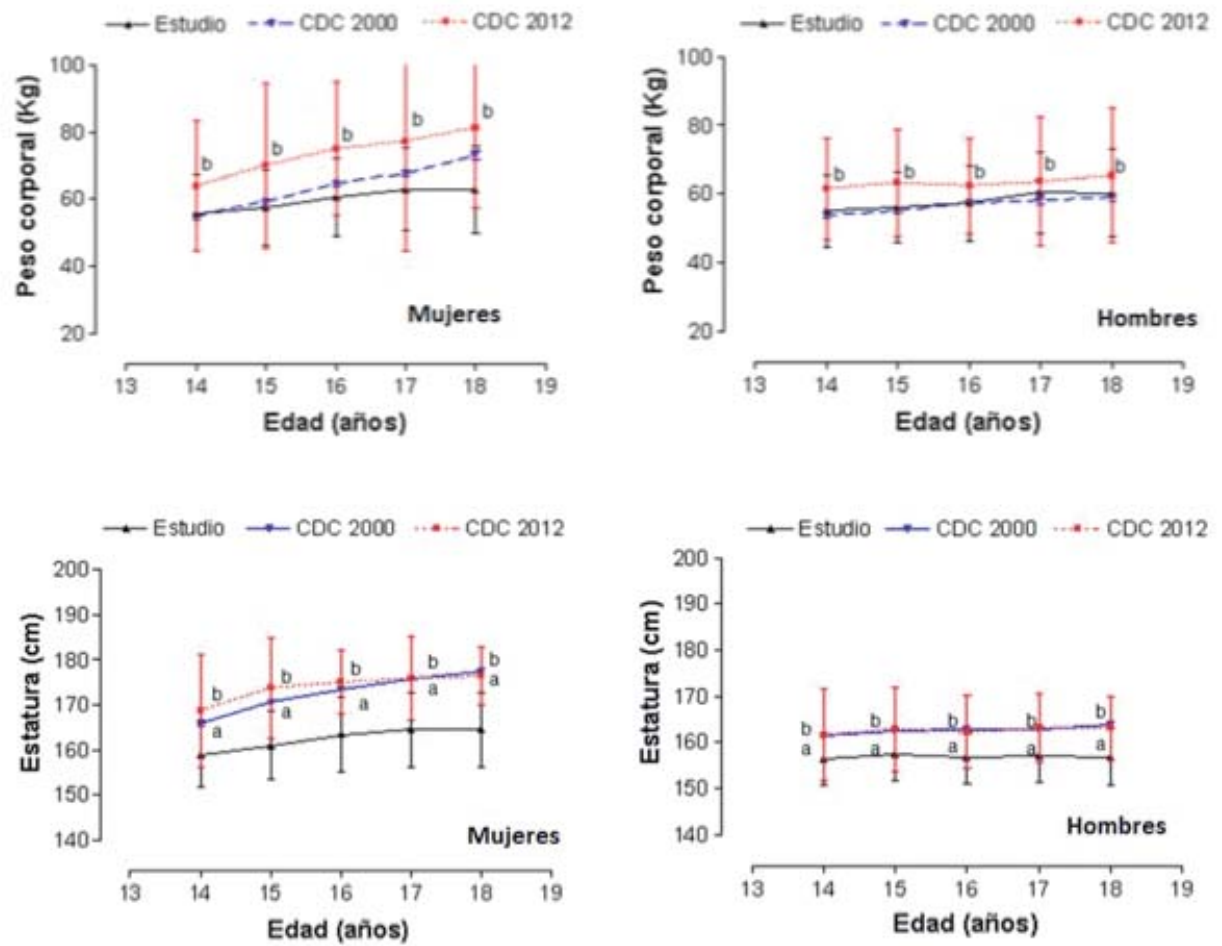

a: diferencia significativa en relación a la referencia de la CDC-2000, b: diferencia significativa en relación a la referencia de la CDC-2012.

\section{FIGURA 2}

Variable de adiposidad del estudio, CDC-2000 y CDC-2012 expresados en promedio y Error Estándar de adolescentes chilenos.
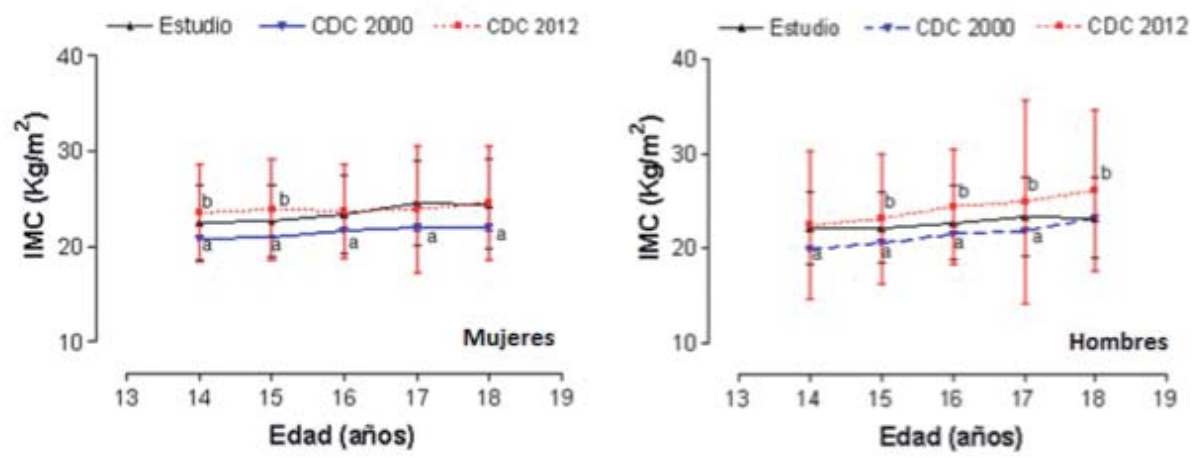

a: diferencia significativa en relación a la referencia de la CDC-2000, b: diferencia significativa en relación a la referencia de la CDC-2012. 
curva del CDC-2000 y próximo a la curva del CDC-2012, sin embargo, esto no ha sido evidenciado en este estudio, lo que pone de manifiesto, que tales escolares no han sufrido aumentos significativos en el peso corporal.

Por otro lado, respecto a la estatura, los resultados muestran que los escolares estudiados presentan menor estatura en relación a ambas referencias (CDC-2000 y CDC-2012). Esto demuestra que los adolescentes chilenos aun muestran baja estatura, a pesar de que en años anteriores se consideraba una clara tendencia a mejorar la estatura promedio de la población chilena en más o menos $1,5 \mathrm{~cm}$ cada 10 años (19). Inclusive en un estudio realizado por Burrows et al (20) en tres regiones de Chile calificaron a los escolares con talla baja su adecuación estatural, considerándolos por debajo de los estándares de la Organización Mundial de la Salud. De hecho, en muchos países latinoamericanos se observó una tendencia secular positiva en la estatura debido a la mejora social y de las condiciones económicas (17), consecuentemente una aproximación y/o superación a los valores medios de las referencias internacionales, respectivamente.

\section{Adiposidad corporal}

La prevalencia de exceso de peso fue determinada a través del Índice de Masa Corporal (IMC). Los resultados muestran que a partir de la referencia del CDC-2000 se observó $28,32 \%$ de exceso de peso en las niñas y 32,47 en los niños. De hecho, varios estudios internacionales muestran valores similares de sobrepeso y obesidad en escolares adolescentes de Argentina (21), Ecuador (22) y México (23), respectivamente. Evidentemente la prevalencia de sobrepeso y obesidad es un problema de salud pública que se presenta no sólo en Chile, sino también en países desarrollados, así como en países en vías de desarrollo (24).

Por otro lado, cuando se realizó el análisis de la prevalencia del exceso de peso por medio de la referencia del CDC-2012 con datos más actualizados y recientes, observamos que las proporciones son extremadamente inferiores, verificándose $6,64 \%$ en las niñas y 9,02\% en los niños. Estos hallazgos por una parte pueden ser interpretados como una baja prevalencia del exceso de peso cuando se compara con una curva reciente, sin embargo, cuando se compara con la curva del CDC-2000 no actualizada, los resultados reflejan altos valores de prevalencia en el exceso de peso. Sin duda, la valoración del crecimiento físico y el estado nutricional deben ser determinadas a través referencias nacionales $y / 0$ internacionales actualizadas, ya que un posible cambio de referencia podría provocar confusión a la hora de analizar el crecimiento físico y el estado nutricional de adolescentes chilenos. En este sentido, nuestros hallazgos son consistentes, puesto que no sólo, es decidir, si, se utiliza o no, una nueva referencia internacional, sino más bien proponer una curva de crecimiento actualizada para niños y adolescentes chilenos, con lo que se podría evitar confusiones de cualquier naturaleza y principalmente de índole secular. De hecho, muchos países han desarrollado sus propios estándares de crecimiento, pero requiere de extensos y costosos estudios (25), aunque una de las ventajas potenciales que ofrece este tipo de investigación es medir el real estado de salud de niños y adolescentes.

En general, los valores medios del IMC del estudio son mayores en relación a la referencia del CDC-2000, sin embargo, los valores medios del CDC-2012 son superiores en las mujeres a los 14 y 15 años y en los hombres desde los 15 hasta los 18 años. De hecho, este patrón se debe a la baja estatura observada en los adolescentes de ambos sexos, puesto que en la relación del peso con la estatura, esta última variable afecta el IMC, por lo que la aplicación de este índice sería inapropiado para personas de baja estatura $(26,27)$, consecuentemente podría sobrestimar el exceso de peso en individuos de pequeña estatura.

El estudio presenta algunas limitaciones, como por ejemplo la falta de control en la calidad de las medidas antropométricas, aunque esto no invalida nuestros resultados, puesto que antes del proceso de recolección de datos, los evaluadores participaron de un programa piloto de entrenamiento. Por otro lado, los hábitos de alimentación no fueron controlados, hecho que podría originar sesgo en los resultados, pero dado el tamaño muestral utilizado en el estudio es posible inferir su posible generalización a la población de adolescentes escolares chilenos, además sugerimos para futuros estudios controlar tales variables y evaluar los parámetros del crecimiento físico en edades más tempranas.

\section{TABLA 3}

Indicadores de adiposidad corporal y prevalencia de bajo y exceso de peso de adolescentes chilenos.

\begin{tabular}{|c|c|c|c|c|c|c|c|c|}
\hline \multirow[b]{2}{*}{ Adiposidad } & \multicolumn{4}{|c|}{ Mujeres $(n=6390)$} & \multicolumn{4}{|c|}{ Hombres $(n=13580)$} \\
\hline & & & & & \multirow{2}{*}{\multicolumn{4}{|c|}{$22,2 \pm 3,8$}} \\
\hline $\mathrm{IMC}\left(\mathrm{Kg} / \mathrm{m}^{2}\right)$ & \multicolumn{4}{|c|}{$22,7 \pm 3,9$} & & & & \\
\hline \multirow[t]{2}{*}{ Categorías (\%) } & \multicolumn{2}{|c|}{ CDC-2000 } & \multicolumn{2}{|c|}{ CDC-2012 } & \multicolumn{2}{|c|}{ CDC-2000 } & \multicolumn{2}{|c|}{ CDC-2012 } \\
\hline & $\mathrm{n}$ & $\%$ & $\mathrm{n}$ & $\%$ & $\mathrm{n}$ & $\%$ & $\mathrm{n}$ & $\%$ \\
\hline Bajo peso & 256 & 4,01 & 567 & 8,88 & 727 & 5,35 & 1202 & 8,85 \\
\hline Eutrófico & 4324 & 67,67 & 5398 & 84,48 & 8444 & 62,18 & 11153 & 82,13 \\
\hline Sobrepeso & 1351 & 21,14 & 382 & 5,98 & 2150 & 15,83 & 1108 & 8,16 \\
\hline Obesidad & 459 & 7,18 & 42 & 0,66 & 2259 & 16,64 & 117 & 0,86 \\
\hline Total & 6390 & 100 & 6390 & 100 & 13580 & 100 & 13580 & 100 \\
\hline Exceso de peso & 1810 & 28,32 & 424 & 6,64 & 4409 & 32,47 & 1225 & 9,02 \\
\hline
\end{tabular}




\section{CONCLUSIÓN}

Se concluye que los escolares adolescentes chilenos mostraron similar peso corporal, menor estatura y altos valores de prevalencia de exceso de peso $(28,3$ a $32,5 \%)$ mediante la referencia del CDC-2000, sin embargo, respecto a la referencia del CDC-2012, los adolescentes chilenos se caracterizan con bajo peso y estatura, consecuentemente permitió mostrar valores inferiores de exceso de peso $(9,0$ a 6,6\%). Los resultados sugieren construir curvas actualizadas para valorar el real crecimiento físico y el estado nutricional de la población escolar chilena y consecuentemente evitar posibles confusiones durante el diagnóstico, vigilancia y seguimiento del estado de salud. Al respecto, Atalah et al (28) consideran que lo ideal es definir mediante una mesa de trabajo sobre qué referencia y que puntos de corte utilizar, para lograr un consenso técnico que sea posteriormente una norma nacional.

\section{RESUMEN}

El objetivo del estudio fue comparar los parámetros del crecimiento físico y de adiposidad corporal en adolescentes chilenos en función a la referencia del CDC-2000 y CDC-2012. Se estudiaron 19970 escolares de 14 a 18 años. Los adolescentes pertenecen a las tres dependencias de escolaridad del sistema educativo chileno. Se determinó el peso, estatura y el Índice de Masa Corporal. Los resultados indicaron que los escolares chilenos mostraron similar peso corporal, menor estatura y altos valores de prevalencia de exceso de peso $(28,3$ a 32,5\%) a través de la referencia del CDC-2000, sin embargo, por medio de la referencia del CDC-2012, mostraron bajo peso y estatura, consecuentemente valores inferiores de exceso de peso $(9,0$ a $6,6 \%)$. Se concluye que el uso de una referencia actualizada puede causar confusión al analizar el crecimiento físico y la adiposidad corporal de adolescentes chilenos. Los resultados sugieren la construcción de una curva nacional, con lo cual, es posible efectuar la valoración del crecimiento y estado nutricional de forma más confiable.

Palabras clave: Crecimiento, adiposidad, adolescentes, curvas de estatura e IMC.

Agradecimientos: Este estudio fue posible gracias al financiamiento del Instituto Nacional del Deporte IND, Chile.

Conflicto de interés: Los autores declaran no tener ningún tipo de conflicto de interés en este artículo.

\section{BIBLIOGRAFÍA}

1. Soares NT. Um novo referencial antropométrico de crescimento: significados e implicações. Rev Nutr 2003;16:93104.

2. Ahn Y, Cho S, Sohn M. Adiposity of Korean School-Age Children Measured by National and International Growth Charts. Res Nurs Health 2013;36:16-25.

3. Ho SY, Lam TH, Janus ED; Hong Kong Cardiovascular Risk Factor Prevalence Study Steering Committee. Waist to stature ratio is more strongly associated with cardiovascular risk factors than other simple anthropometric indices. Ann Epidemiol. 2003;13(10):683-91.

4. Núñez-Rivas $H P$, Monge-Rojas $R$, León $H$, Roselló $M$. Prevalence of overweight and obesity among Costa Rican elementary school children. Rev Panam Salud Publica. 2003;13(1):24-32.

5. Lobstein T, Baur L, Uauy R; IASO International Obesity TaskForce. Obesity in children and young people: a crisis in public health. Obes Rev. 2004;5 Suppl 1:4-104.
6. Muzzo S. Crecimiento normal y patológico del niño y del adolescente. Rev Chil Nutr. 2003;30(2):92-100.

7. Cossio-Bolaños MA, Arruda M, Núñez Álvarez V, Lancho Alonso JL. Efectos de la altitud sobre el crecimiento físico en niños y adolescentes. Rev Andal Med Deporte 2011;4 (2):71-6.

8. Cossio-Bolaños MA, Pablos Abella C, Arruda M. Valoración de la adiposidad corporal de escolares en Arequipa, Perú. Rev Peru Med Exp Salud Publica. 2012;29(4):477-82.

9. World Health Organization. Multicentre Growth Reference Study Group. Assessment of differences in linear growth among populations in the WHO Multicentre Growth Reference Study. Acta Paediatr Suppl 2006;450:56-65.

10. Kuczmarski R, Ogden C, Grummer-Strawn L, et al: CDC growth charts: United States. Advance data from vital and health statistics. Hyattsville (MD): U.S. Department of Health and Human Services; 2000.

11. Katzmarzyk PT. Waist circumference percentiles for Canadian youth 11-18y of age. European J Clin Nutr. 2004; 58: 1011-5.

12. Fryar CD, Gu Q, Ogden CL. Anthropometric reference data for children and adults: United States, 2007-2010. National Center for Health Statistics. Vital Health Stat 11(252). 2012.

13. Cole TJ, Bellizzi MC, Flegal M, Dietz WH. Establishing a standard definition for child overweight and obesity worldwide: international survey. Br Med J. 2000; 320: 1240-3.

14. Sistema de Medición de la Calidad de la Educación del Ministerio de Educación de Chile (SIMCE, 20012). Informe de resultados de educación física $8^{\circ}$ básico, Ministerio de Educación, Unidad de currículum y evaluación, Santiago, 2011.

15. Centers for Disease Control and Prevention. National Health and Nutrition Examination Survey (NHANES) anthropometry procedures manual. 2009. Available from: http://www.cdc.gov/nchs/data/nhanes/nhanes_09_10/ BodyMeasures_09.pdf.

16. Garrow JS, Webster J. Quetelet's index (WIH2) as a measure of fatness. Int J Obes 1985;9(2):147-53.

17. Olszowy Km, Dufour DL, Bender RI, Bekelman TA. And Reina JC. socioeconomic status, Stature, and Obesity in Women: 20-Year Trends in Urban Colombia. Am J Hum Biol. 2012;24:602-10.

18. Du S, Lu B, Zhai F, Popkin BM. A new stage of the nutrition transition in China. Public Health Nutr. 2002; 5 (1A): 169-74.

19. Minsal. Situación de Seguridad Alimentaria en Chile. Plan Nacional de Acción en Nutrición. Comisión Nacional de Alimentación y Nutrición 1996: p 61-70.

20. Burrows A, Ramírez I, Cordero J, Muzzo S. Cambio secular del retraso de talla en escolares chilenos de tres regiones del país. Rev Chil Pediatr. 1999;.70(5):390-7.

21. Kovalskys I, Bay L, Rausch HC, Berner E. Prevalencia de obesidad en una población de 10 a 19 años en la consulta pediátrica. Rev Chil Pediatr. 2005, vol.76, n.3, 324-5.

22. Yepez R, Carrasco F, Baldeón ME. Prevalencia de sobrepeso $y$ obesidad en estudiantes adolescentes ecuatorianos del área urbana. Arch Latinoam Nutr. 2008;58( 2):139-43.

23. Ortiz Hernández L. Estado nutricio en adolescentes de una población suburbana de la ciudad de México. Rev Mex Pediatr. 2003; 70(3); 109-17.

24. Chescheir N. Obesidad en el Mundo y su Efecto en la Salud de la Mujer. Obstet Gynecol. 2011; 117: 1213-22. 
25. Silva S, Maia J, Claessens AL, Beunen G, Pan H. Growth references for Brazilian children and adolescents: Healthy growth in Cariri study. Ann Hum Biol. 2012; 39(1): 11-8.

26. Lara-Esqueda A, Aguilar-Salinas CA, Velazquez-Monroy $O$, Gómez-Pérez FJ, Rosas-Peralta M, Mehta R et al. The body mass index is a less-sensitive tool for detecting cases with obesity-associated co-morbidities in short stature subjects. Int J Obes Relat Metab Disord. 2004; 28: 1443-50.
27. Cossio-Bolaños MA, Maria TS; Campos RG; Pascoal EH; Eduardo-Hespanhol J; Arruda M. O uso das curvas de crescimento da Organização Mundial da Saúde em crianças e adolescentes que vivem em regiões de altitude moderada. Rev Paul Pediatr. 2012;30(3):314-20.

28. Atalah S, Loaiza S, Taibo M. Estado nutricional en escolares chilenos según la referencia NCHS y OMS 2007. Nutr Hosp. 2012;27(1):1-6. 